\title{
Psychosocial factors are preventive against coronary events in Japanese men with coronary artery disease: The Eastern Collaborative Group Study 7.7-year follow-up experience
}

\author{
Reiko Hori ${ }^{{ }^{*+}}$, Jun-ichiro Hayano ${ }^{2+}$, Kazuhiro Kimura ${ }^{3+}$, Nitaro Shibata ${ }^{4+}$ and Fumio Kobayashi ${ }^{1+}$
}

\begin{abstract}
Background: The Japanese Coronary-prone Behaviour Scale (JCBS) is a questionnaire developed by the Eastern Collaborative Group Study (ECGS), a multi-centre study of coronary-prone behaviour among Japanese men. Subscale C of the JCBS consists of 9 items that have been independently associated with the presence of coronary artery disease (CAD) in patients undergoing coronary angiography (CAG). There have been no reports of a relationship between any behavioural factor and the prognosis of CAD in Japan. The purpose of the current study was to investigate behavioural correlations with the prognosis of CAD as a part of the ECGS.
\end{abstract}

Methods: We examined the mortality and coronary events of 201 men ( $58 \pm 10,27-86$ years) enrolled in the ECGS from 1990 to 1995, who underwent diagnostic coronary angiography and were administered the JCBS and the Japanese version of the Jenkins Activity Survey (JAS) Form C. Their health information after CAG was determined by a review of their medical records and by telephone interviews that took place from 2002 to 2003.

Results: Cardiac events during the follow-up period ( $7.7 \pm 4.2$ years) included 13 deaths from CAD, 25 cases of new-onset myocardial infarction, 26 cases of percutaneous coronary intervention, and 19 cases of coronary artery bypass graft surgery. There was no difference in established risk factors between groups with and without cardiac events. Seven factors were extracted by principal component analysis in order to clarify which factors were measured by the JCBS. Stepwise multivariate Cox-hazard regression analysis, in which 9 standard coronary risk factors were forced into the model, showed that Factor 4 from the JCBS (namely, the Japanese spirit of 'Wa') was independently associated with coronary events (hazard ratio: $0.21 ; p=0.01$ ). By other Cox-hazards regression analyses of coronary events using each set of JAS scores and the JCBS Scale C score instead of Factor 4 as selectable variables, the JAS scores or the JCBS Scale $C$ score were not entered into the models.

Conclusion: The Japanese spirit of ' Wa' is a preventive factor against coronary events for Japanese men with CAD.

Keywords: Coronary artery disease, Prognosis, Risk, Behavioural medicine, Psychosocial factor, Japanese

\footnotetext{
* Correspondence: rhori@aichi-med-u.ac.jp

${ }^{\dagger}$ Equal contributors

'Department of Health and Psychosocial Medicine, Aichi Medical University

School of Medicine, 1-1 Yazako Karimata, Nagakute, Aichi 480-1195, Japan

Full list of author information is available at the end of the article
}

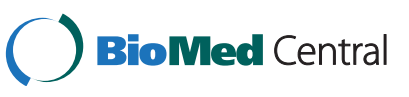

(c) 2015 Hori et al.; licensee BioMed Central. This is an Open Access article distributed under the terms of the Creative Commons Attribution License (http://creativecommons.org/licenses/by/4.0), which permits unrestricted use, distribution, and reproduction in any medium, provided the original work is properly credited. The Creative Commons Public Domain Dedication waiver (http://creativecommons.org/publicdomain/zero/1.0/) applies to the data made available in this article, unless otherwise stated. 


\section{Background}

Psychosocial factors are recognized as important risk factors for cardiovascular disease (CAD). What has been called the 'Type A' behaviour pattern has been described as a coronary-prone behavioural type in Western studies. This pattern is characterized by attributes such as hard-driving effort, striving for achievement, competitiveness, aggressiveness, haste, impatience, restlessness, alertness, uneven bursts of amplitude in speech, and hurried motor movements. Individuals who exhibit this behaviour pattern are usually conscientiously committed to, and often successful in, their occupations [1]. The Secondary Prevention in Uppsala Primary Health Care Project [2] has demonstrated the effectiveness of cognitive behavioural therapy focused on stress management, coping with stress, and reducing experiences of daily stress, time urgency, and hostility in CAD patients. However, the impact and content of risk are thought to differ by geographic region, ethnic origin, and society. In the INTERHEART study [3], general stress and depression in acute myocardial patients had different odds ratios by region and ethnic group. Coronary-disease-prone behaviour in Japan is thought to be characterized by less aggression and a greater tendency to display 'workaholic' characteristics than that in Western countries [4].

The Eastern Collaborative Group Study (ECGS) [5] is a multi-centre study in Japan aimed at investigating behavioural correlates of CAD among contemporary Japanese people, beyond the concept of Type A behaviour patterns. In this project, we developed a questionnaire called the Japanese Coronary-prone Behaviour Scale (JCBS), which consists of 122 questions that evaluate 10 behavioural and psychological features: 1) attitude toward one's job, 2) psychophysiological characteristics, 3) speed and impatience, 4) eating behaviour, 5) style of speech, 6) Japanese mentality, 7) stylistic hostility, 8) emotional hostility, 9) social support, and 10) developmental history [4]. It incorporates items beyond the concept of Type A behaviour patterns, and includes behavioural characteristics specific to Japanese society and cultures. A previous study [6] using the JCBS showed that 9 items from the JCBS, named Scale $C$, were independently associated with the presence of $\mathrm{CAD}$ in patients undergoing coronary angiography (CAG). Scale C represents job-centred lifestyle, social dominance, and suppressed overt Type A behaviour. The external validity of the JCBS Scale $C$ was confirmed by a separate study [7] using different participants.

Although low social support and negative emotions, such as depression, have been shown to aggravate the prognosis of CAD patients in Western countries [8-24], to our knowledge there is no report that clarifies how psychosocial risk factors are related to the prognosis of Japanese CAD patients. The purpose of this study was to investigate behavioural factors predicting the prognosis of Japanese men with established CAD.

\section{Methods \\ Participants}

Participants were 201 men of the initial ECGS who had $\mathrm{CAD}$ at the index angiogram performed between 1990 and 1995. They were admitted to the hospital one or two days before the CAG. Medical histories and status of coronary risk factors, including hypertension, diabetes mellitus, smoking, and obesity, were obtained on admission day 1 from medical records. Levels of plasma lipids after $>14$ hours of fasting were assessed on admission day 2. Participants were administered the JCBS and the Japanese version of the Jenkins Activity Survey (JAS) Form $\mathrm{C}$ while undergoing diagnostic CAG.

The JAS was developed in an attempt to duplicate the clinical assessment of the Type A behaviour pattern by employing an objective psychometric procedure. The JAS is a self-administered, computer-scored questionnaire that was constructed in an effort to develop a quicker, less expensive, more uniform, and better-calibrated procedure for judging coronary-prone behaviour patterns in large groups of participants [1]. A simple scoring method applied to this test validly identified the behaviour patterns of approximately $72 \%$ of a sample of men; the standardized interview was the criterion [1]. Since this initial step toward validation, more sophisticated scaling and scoring procedures have been developed. These have, in turn, been applied retrospectively to a sample of men with coronary heart disease. The JAS has been revised several times and the result is the JAS Form $\mathrm{C}$, which is the version typically used. The JAS yields a composite Type A scale score and three factor-analytically-derived subscales: Speed and impatience (Factor S), Job involvement (Factor J), and Hard-driving and competitive (Factor C).

Participants' health information after CAG was determined by a review of medical records and telephone interviews from 2002 to 2003. Primary end points were new-onset cardiac events, including cardiac death, angina pectoris, acute myocardial infarction (AMI), percutaneous coronary intervention (PCI), and coronary artery bypass graft (CABG).

This study's protocol was approved by the Ethical Committee of Aichi Medical University School of Medicine.

\section{Statistical analysis}

The SAS program (SAS Institute, Cary, NC) was used for statistical analysis. Differences in quantitative variables between the two groups were analysed with the Student's $t$-test, and differences in categorical data were analysed with the chi-square test. 
Principal component analysis with promax rotations was used to confirm which factors were measured by the JCBS, the questions of which were originally based on behavioural and psychological features, because the abstracted factors correlated with each other: -0.52 for Factors 1 and 6, 0.46 for Factors 3 and 6, 0.51 for Factors 3 and $7,-0.41$ for Factors 4 and 5 , and 0.44 for Factors 4 and 7 . The scree test was used to determine the number of factors to retain in the principal component analysis.

Stepwise multivariate Cox-hazard regression stratified by medical centre was used for evaluating the independent effect of the JCBS factors and standard coronary risks factors. Standard coronary risk factors, including age, body mass index, HDL cholesterol, LDL cholesterol, triglycerides, current smoking habits, severity of coronary stenosis, and history of diabetes and hypertension at the first admission, were forced into this regression model. Continuous values were used for age, body mass index, HDL cholesterol, LDL cholesterol, triglycerides, and the number of coronary artery branches involved; categorical values were used for current smoking habits (i.e. current smoker or not) and presence of diabetes and hypertension. The number of coronary artery branches involved was defined as the number of major coronary arteries or branches with at least one clinically significant stenosis whose luminal narrowing was equal to or more than $50 \%$, or with a lesion that had been the subject of intervention. Clinically significant stenosis in the left main coronary artery was defined as three-vessel stenosis. The presence of hypertension was determined if the subject was under treatment or had equal to or more than $160 \mathrm{~mm}$ mercury of systolic blood pressure or equal to or more than $90 \mathrm{~mm}$ mercury of diastolic blood pressure on admission. The presence of diabetes was determined if the subject was under treatment or the result of an oral glucose tolerance test met the criteria of diabetes. This analysis was performed after stratifying by hospital because the patients who participated were initially drawn from nine hospitals throughout the country.

\section{Results}

During the follow-up period ( $7.7 \pm 4.2$ years), 23 (11\%) men died, $13(6 \%)$ of whom died from cardiovascular disease. AMI or angina occurred in 25 (12\%) cases, PCI in 26 (13\%), and CABG in 19 (9\%). Patients with cardiac death, new-onset AMI or angina, PCI, or CABG were counted in the event group. The primary characteristics of patients grouped by cardiac event are shown in Table 1. There were no differences in standard risk factors, JAS scores, or the JCBS Scale C score between groups with and without events.

Seven factors were extracted by principal component analysis to clarify which factors were measured by the
Table 1 Characteristics of patients grouped by cardiac events

\begin{tabular}{lccc}
\hline & Event (-) (138) & Event (+) (63) & $\boldsymbol{p}$ value \\
\hline Age & $59 \pm 9$ & $58 \pm 10$ & $\mathrm{~ns}$ \\
LDL cholesterol (mg/dl) & $131 \pm 33$ & $138 \pm 38$ & $\mathrm{~ns}$ \\
Triglycerides (mg/dl) & $153 \pm 91$ & $151 \pm 112$ & $\mathrm{~ns}$ \\
HDL cholesterol ( mg/dl) & $38 \pm 11$ & $37 \pm 9$ & $\mathrm{~ns}$ \\
Body mass index (kg/m²) & $23 \pm 2$ & $24 \pm 2$ & $\mathrm{~ns}$ \\
Diabetes mellitus (\%) & 22 & 19 & $\mathrm{~ns}$ \\
Hypertension (\%) & 41 & 51 & $\mathrm{~ns}$ \\
Smoking habits (\%) & 29 & 32 & $\mathrm{~ns}$ \\
Number of coronary artery & $2 \pm 1$ & $2 \pm 1$ & $\mathrm{~ns}$ \\
branches involved & & & \\
JAS Type A score & $-0.2 \pm 8.8$ & $-0.6 \pm 8.0$ & $\mathrm{~ns}$ \\
$\quad$ Factor S score & $-4.3 \pm 9.2$ & $-3.6 \pm 9.2$ & $\mathrm{~ns}$ \\
$\quad$ Factor J score & $-11.1 \pm 7.8$ & $-12.9 \pm 9.0$ & $\mathrm{~ns}$ \\
$\quad$ Factor H score & $-2.0 \pm 10.0$ & $-0.2 \pm 11.2$ & $\mathrm{~ns}$ \\
JCBS Scale C score & $0.24 \pm 1.07$ & $0.08 \pm 1.07$ & $\mathrm{~ns}$ \\
\hline
\end{tabular}

JAS = Jenkins Activity Survey, $\mathrm{HDL}=$ high-density lipoprotein, $\mathrm{LDL}=$ low-density lipoprotein, and JCBS = Japanese Coronary-Prone Behaviour Scale. 'Event' includes cardiac deaths and new onset of $\mathrm{AMI}$ or angina, $\mathrm{PCl}$, and $C A B G$.

JCBS (Table 2). Since the scree test showed that the smooth decrease of eigenvalues appeared to level off to the right of the $7^{\text {th }}$ plot, we extracted seven factors.

The results of Cox-hazards regression analysis of coronary events with JCBS factors and standard coronary risks are shown in Table 3. Nine standard coronary risk factors were forced into the model, and HDL cholesterol was identified as a significant predictor of the recurrence of cardiovascular events. In that model, Factor 4 on the JCBS was extracted as a significant and independent predictor. By other similar Cox-hazards regression analyses of coronary events using each set of JAS scores and the JCBS Scale C score instead of Factor 4 as selectable variables and after 9 standard coronary risk factors were forced into the model, the JAS scores or the JCBS Scale $\mathrm{C}$ score were not entered into the models (not shown).

\section{Discussion}

This follow-up study of Japanese men with established CAD showed that the fourth factor of the JCBS had a protective effect against cardiovascular events. This is the first follow-up study in which the relationship between behavioural factors and CAD prognosis was examined among Japanese male patients.

Contrary to findings in other countries, a prospective analysis in a Japanese population shows that lower levels of impatience were associated with a 1.4-fold higher multivariable-adjusted risk of incidence of coronary heart disease and a 1.5-fold higher multivariable-adjusted risk of incidence of myocardial infarction and non-fatal coronary 
Table 2 Factor loading of each item and factor of the JCBS

\begin{tabular}{|c|c|c|c|c|c|c|c|}
\hline & $\begin{array}{l}\text { Factor } 1 \\
\text { (Workaholic) }\end{array}$ & $\begin{array}{l}\text { Factor } 2 \\
\text { (Speed and } \\
\text { impatience) }\end{array}$ & $\begin{array}{l}\text { Factor } 3 \\
\text { (Social } \\
\text { support) }\end{array}$ & $\begin{array}{l}\text { Factor } 4 \\
\text { (Japanese spirit of 'Wa'; } \\
\text { harmonious groupism) }\end{array}$ & $\begin{array}{l}\text { Factor } 5 \\
\text { (Happy childhood } \\
\text { memories) }\end{array}$ & $\begin{array}{l}\text { Factor } 6 \\
\text { (Undesirable } \\
\text { dietary style) }\end{array}$ & $\begin{array}{l}\text { Factor } 7 \\
\text { (Independent } \\
\text { personality) }\end{array}$ \\
\hline JCBS 7 & 0.6657 & -0.01544 & -0.05889 & 0.03374 & -0.06228 & -0.05411 & 0.00081 \\
\hline JCBS 2 & 0.66939 & -0.07549 & 0.04187 & 0.02992 & 0.04105 & -0.16959 & 0.02506 \\
\hline JCBS 4 & 0.70306 & -0.00579 & 0.17555 & 0.02065 & 0.12309 & 0.02082 & -0.1754 \\
\hline JCBS 11 & 0.69264 & 0.12182 & 0.06037 & -0.0468 & 0.16823 & 0.15507 & -0.10799 \\
\hline JCBS 8 & 0.5968 & -0.04097 & 0.06928 & 0.02429 & -0.04449 & 0.00047 & -0.02594 \\
\hline JCBS 12 & 0.644 & 0.1912 & 0.18932 & -0.00729 & 0.13311 & 0.04228 & 0.00158 \\
\hline JCBS 1 & 0.58644 & 0.11522 & 0.17882 & -0.1636 & 0.1866 & -0.07635 & 0.15225 \\
\hline JCBS 5 & 0.52427 & -0.16922 & 0.24316 & -0.02231 & 0.07559 & 0.09161 & 0.17102 \\
\hline JCBS 61 & 0.45019 & 0.19799 & 0.04548 & -0.05218 & 0.22504 & -0.18192 & 0.1344 \\
\hline JCBS 53 & 0.4999 & 0.18488 & 0.26705 & -0.21271 & 0.18524 & -0.08068 & 0.11102 \\
\hline JCBS 9 & 0.48181 & 0.10895 & 0.44359 & -0.01062 & 0.14866 & 0.07239 & 0.26366 \\
\hline JCBS 79 & 0.38811 & 0.36497 & 0.06964 & 0.09301 & -0.08315 & 0.02752 & -0.15673 \\
\hline JCBS 59 & 0.40289 & 0.28582 & 0.09261 & 0.05008 & 0.16766 & -0.18976 & 0.29137 \\
\hline JCBS 66 & 0.41422 & 0.30333 & 0.36356 & 0.32523 & 0.04684 & 0.18392 & 0.06059 \\
\hline JCBS 86 & 0.33111 & 0.28684 & 0.158 & 0.06694 & -0.06065 & 0.12592 & 0.04206 \\
\hline JCBS 20 & 0.15001 & 0.02207 & 0.00083 & 0.10009 & -0.06369 & -0.01951 & -0.07811 \\
\hline JCBS 19 & -0.33296 & 0.28941 & -0.08421 & 0.16522 & -0.23582 & 0.09002 & 0.00166 \\
\hline JCBS 26 & -0.07569 & 0.50125 & -0.09204 & 0.12555 & -0.07603 & 0.22705 & -0.17038 \\
\hline JCBS 27 & -0.06801 & 0.51653 & 0.15822 & 0.16726 & -0.01501 & 0.25978 & 0.00435 \\
\hline JCBS 25 & 0.13943 & 0.44699 & 0.09794 & 0.03393 & 0.03305 & 0.02621 & 0.05655 \\
\hline JCBS 18 & -0.05127 & 0.45742 & -0.15281 & 0.25542 & -0.13299 & 0.08046 & -0.02773 \\
\hline JCBS 23 & 0.22695 & 0.42874 & -0.10483 & -0.02407 & 0.01333 & 0.03807 & 0.03509 \\
\hline JCBS 22 & 0.29365 & 0.41337 & -0.12816 & -0.03467 & 0.03566 & 0.14669 & -0.07292 \\
\hline JCBS 46 & -0.00788 & 0.46771 & 0.04789 & 0.20904 & -0.14638 & 0.23808 & 0.20028 \\
\hline JCBS 30 & -0.00656 & 0.43062 & -0.03661 & 0.15581 & 0.00808 & 0.16003 & 0.12011 \\
\hline JCBS 45 & -0.13756 & 0.43712 & -0.18272 & 0.30337 & -0.35336 & 0.279 & -0.07923 \\
\hline JCBS 94 & -0.17995 & 0.4275 & -0.16184 & 0.36802 & -0.08971 & -0.11907 & 0.07832 \\
\hline JCBS 44 & -0.18733 & 0.45537 & -0.08459 & 0.40844 & -0.43417 & 0.3293 & 0.04604 \\
\hline JCBS 52 & 0.11414 & 0.38368 & 0.15322 & 0.11326 & -0.06257 & 0.12267 & -0.16308 \\
\hline JCBS 24 & 0.31927 & 0.37819 & 0.15927 & -0.09052 & 0.15054 & -0.12432 & 0.15631 \\
\hline JCBS 67 & 0.21982 & 0.36894 & 0.09766 & -0.10005 & 0.03008 & -0.01498 & 0.2324 \\
\hline JCBS 42 & 0.16575 & 0.4495 & 0.23697 & 0.31087 & 0.03301 & 0.16148 & 0.1241 \\
\hline JCBS 95 & -0.13882 & 0.39013 & -0.15219 & 0.25738 & -0.18216 & -0.14097 & 0.20038 \\
\hline JCBS 43 & -0.20393 & 0.36447 & -0.16696 & 0.35761 & -0.15165 & 0.08106 & -0.20775 \\
\hline JCBS 88 & 0.03973 & 0.38072 & 0.06979 & 0.23257 & 0.1357 & -0.07148 & 0.19152 \\
\hline JCBS 81 & 0.0367 & 0.34333 & 0.14592 & 0.15006 & 0.12468 & -0.05457 & 0.11338 \\
\hline JCBS 85 & 0.3001 & 0.32621 & 0.12335 & -0.0024 & 0.18982 & 0.04389 & -0.05015 \\
\hline JCBS 17 & -0.0318 & 0.34856 & -0.10023 & 0.21135 & -0.20494 & 0.08518 & 0.05008 \\
\hline JCBS 97 & 0.07408 & 0.33041 & 0.17667 & 0.02562 & -0.0243 & 0.30175 & 0.04942 \\
\hline JCBS 64 & 0.37515 & 0.34738 & 0.22695 & 0.00772 & 0.11119 & -0.02925 & 0.11571 \\
\hline JCBS 21 & 0.09574 & 0.32233 & 0.0388 & 0.20347 & -0.04849 & -0.02085 & 0.04544 \\
\hline JCBS 28 & 0.01861 & 0.28492 & -0.09643 & 0.04103 & -0.08577 & 0.04365 & 0.1485 \\
\hline JCBS 14 & 0.06726 & 0.24228 & -0.07494 & -0.05824 & 0.01332 & 0.08917 & -0.1116 \\
\hline
\end{tabular}


Table 2 Factor loading of each item and factor of the JCBS (Continued)

\begin{tabular}{|c|c|c|c|c|c|c|c|}
\hline JCBS 77 & 0.17434 & 0.29623 & -0.08518 & 0.13654 & 0.00409 & 0.01672 & 0.01262 \\
\hline JCBS 41 & -0.08561 & 0.26419 & 0.19067 & 0.06778 & -0.05445 & 0.01807 & 0.18801 \\
\hline JCBS 36 & -0.04394 & 0.15025 & -0.01405 & -0.1442 & -0.02217 & -0.00058 & -0.17125 \\
\hline JCBS 90 & 0.06846 & -0.09023 & 0.64526 & -0.0887 & 0.15949 & 0.02903 & -0.03107 \\
\hline JCBS 91 & -0.00203 & 0.0599 & 0.58133 & 0.02309 & 0.24866 & -0.05306 & 0.06609 \\
\hline JCBS 89 & 0.29622 & -0.08047 & 0.63338 & 0.1546 & 0.15514 & 0.08962 & 0.07718 \\
\hline JCBS 96 & 0.01167 & -0.10475 & 0.55056 & -0.18646 & 0.18498 & 0.00671 & 0.05546 \\
\hline JCBS 92 & -0.00858 & 0.01325 & 0.51722 & -0.07883 & 0.04688 & 0.02208 & -0.09845 \\
\hline JCBS 100 & 0.2134 & -0.06753 & 0.53014 & -0.07476 & 0.13151 & 0.08802 & -0.04769 \\
\hline JCBS 93 & 0.17705 & -0.08125 & 0.52357 & 0.03586 & 0.12482 & 0.04661 & 0.17957 \\
\hline JCBS 99 & 0.02482 & -0.02509 & 0.4287 & -0.13756 & 0.17436 & 0.2346 & -0.1314 \\
\hline JCBS 87 & 0.4469 & 0.00565 & 0.48002 & -0.18349 & 0.21304 & -0.11811 & -0.13008 \\
\hline JCBS 56 & 0.26457 & 0.11603 & 0.44431 & -0.22329 & 0.1752 & -0.0467 & -0.04272 \\
\hline JCBS 48 & 0.36848 & 0.15436 & 0.42301 & -0.02913 & 0.26516 & -0.11816 & -0.03833 \\
\hline JCBS 57 & 0.11451 & 0.2262 & 0.32766 & -0.01572 & 0.18355 & 0.02246 & -0.02381 \\
\hline JCBS 35 & -0.06516 & 0.04711 & 0.20655 & 0.15855 & -0.10591 & -0.13739 & 0.07 \\
\hline JCBS 114 & 0.20497 & 0.2254 & 0.29093 & 0.01448 & 0.20263 & 0.03842 & 0.01016 \\
\hline JCBS 112 & -0.07352 & -0.00416 & -0.22813 & -0.0033 & 0.11824 & 0.0983 & 0.02282 \\
\hline JCBS 10 & 0.29624 & 0.15131 & -0.25948 & -0.06458 & 0.04414 & 0.20201 & -0.0644 \\
\hline JCBS 50 & 0.08181 & 0.22404 & -0.16142 & 0.59188 & 0.01463 & 0.01825 & -0.15079 \\
\hline JCBS 55 & -0.02463 & 0.06693 & 0.03597 & 0.56345 & -0.01025 & 0.18295 & -0.07541 \\
\hline JCBS 54 & -0.01411 & 0.19381 & 0.08163 & 0.54285 & -0.17434 & 0.15345 & -0.12387 \\
\hline JCBS 75 & -0.06644 & 0.19038 & 0.04919 & 0.53616 & -0.21321 & 0.11027 & 0.17239 \\
\hline JCBS 49 & 0.20303 & 0.0343 & 0.01538 & 0.41193 & -0.02118 & 0.06043 & -0.22381 \\
\hline JCBS 65 & 0.28458 & 0.23561 & 0.10726 & 0.4714 & -0.0207 & 0.15894 & 0.20435 \\
\hline JCBS 47 & -0.12532 & 0.11299 & -0.16314 & 0.47509 & -0.13236 & 0.10697 & 0.08291 \\
\hline JCBS 78 & 0.01294 & -0.229 & 0.28982 & 0.3523 & 0.0312 & -0.02967 & -0.06426 \\
\hline JCBS 73 & -0.00245 & 0.1842 & -0.0105 & 0.46701 & -0.15905 & 0.16679 & 0.23514 \\
\hline JCBS 62 & 0.2086 & 0.13547 & 0.01018 & 0.37034 & 0.09751 & 0.29022 & 0.06696 \\
\hline JCBS 80 & -0.13451 & 0.34139 & -0.27209 & 0.42403 & -0.05445 & -0.22374 & 0.16423 \\
\hline JCBS 68 & -0.21354 & 0.22512 & -0.16348 & 0.34035 & 0.00638 & -0.19272 & 0.29004 \\
\hline JCBS 29 & -0.06933 & 0.17168 & -0.07026 & 0.26516 & -0.10025 & 0.11571 & -0.04141 \\
\hline JCBS 16 & 0.00698 & 0.11539 & -0.09249 & 0.13222 & -0.06206 & -0.00606 & 0.01106 \\
\hline JCBS 58 & 0.21747 & 0.02575 & -0.08878 & -0.24377 & 0.22553 & -0.18739 & 0.19668 \\
\hline JCBS 117 & 0.20268 & -0.04706 & 0.23214 & -0.10968 & 0.72265 & -0.11323 & 0.0042 \\
\hline JCBS 107 & 0.12388 & -0.01471 & 0.06013 & -0.08219 & 0.59764 & -0.02335 & 0.01895 \\
\hline JCBS 104 & 0.0409 & 0.00942 & 0.21595 & -0.09466 & 0.59397 & 0.11561 & 0.10846 \\
\hline JCBS 122 & -0.02584 & -0.04325 & 0.19593 & 0.16139 & 0.5451 & 0.01578 & 0.23426 \\
\hline JCBS 111 & 0.14297 & 0.17507 & 0.20367 & -0.03662 & 0.44208 & 0.21147 & 0.0175 \\
\hline JCBS 118 & 0.27392 & 0.00152 & 0.40005 & -0.07542 & 0.43322 & 0.0299 & 0.06302 \\
\hline JCBS 121 & -0.03252 & -0.06697 & 0.05893 & 0.06374 & 0.30071 & -0.07693 & 0.14794 \\
\hline JCBS 72 & -0.00927 & -0.00648 & 0.06305 & -0.16514 & 0.25955 & -0.1467 & -0.05453 \\
\hline JCBS 76 & 0.21002 & 0.08095 & 0.23713 & -0.00795 & 0.21793 & 0.12333 & 0.01105 \\
\hline JCBS 102 & 0.04736 & 0.18578 & 0.03297 & 0.19739 & -0.16773 & -0.07515 & 0.18822 \\
\hline JCBS 40 & -0.05862 & 0.22017 & 0.09371 & 0.27771 & -0.22501 & 0.10568 & 0.1409 \\
\hline
\end{tabular}


Table 2 Factor loading of each item and factor of the JCBS (Continued)

\begin{tabular}{|c|c|c|c|c|c|c|c|}
\hline JCBS 120 & -0.07915 & 0.08305 & -0.15092 & 0.24102 & -0.52934 & 0.15085 & 0.08448 \\
\hline JCBS 119 & -0.10988 & 0.08002 & -0.15743 & 0.11462 & -0.68387 & 0.10537 & 0.06965 \\
\hline JCBS 31 & 0.11864 & 0.25764 & 0.13626 & 0.12208 & 0.04839 & 0.52558 & -0.0507 \\
\hline JCBS 38 & -0.09595 & 0.13673 & -0.12985 & 0.20347 & -0.09528 & 0.40525 & 0.03978 \\
\hline JCBS 34 & -0.08502 & 0.14654 & -0.0224 & 0.20199 & 0.01751 & 0.37147 & 0.16665 \\
\hline JCBS 113 & -0.0371 & 0.08721 & -0.05023 & 0.37954 & -0.05483 & 0.38486 & 0.06201 \\
\hline JCBS 106 & 0.01366 & -0.01016 & 0.14024 & 0.17802 & -0.01568 & 0.37374 & -0.15778 \\
\hline JCBS 109 & 0.08942 & 0.29069 & 0.11004 & 0.19584 & -0.24665 & 0.39505 & -0.0259 \\
\hline JCBS 74 & 0.06573 & 0.25493 & -0.00485 & 0.01242 & -0.08814 & 0.31184 & 0.30365 \\
\hline JCBS 98 & 0.03564 & 0.12135 & 0.28263 & -0.03918 & 0.07747 & 0.30424 & -0.25118 \\
\hline JCBS 116 & 0.06314 & 0.10832 & 0.19526 & 0.15951 & -0.00241 & 0.30146 & -0.19585 \\
\hline JCBS 110 & 0.27577 & 0.15513 & 0.29255 & 0.07707 & -0.05192 & 0.29194 & 0.08385 \\
\hline JCBS 108 & 0.01961 & 0.22419 & 0.05044 & 0.1961 & -0.2488 & 0.30021 & 0.06376 \\
\hline JCBS 84 & 0.03769 & -0.12947 & 0.21008 & 0.05492 & -0.01254 & 0.26767 & -0.16522 \\
\hline JCBS 37 & -0.07552 & 0.12013 & 0.00512 & 0.13458 & 0.00274 & 0.24435 & -0.09291 \\
\hline JCBS 105 & -0.06563 & 0.06894 & -0.08361 & -0.02213 & -0.02628 & 0.22403 & -0.01352 \\
\hline JCBS 33 & -0.04418 & 0.0593 & 0.05493 & 0.09348 & -0.17229 & 0.21418 & 0.03581 \\
\hline JCBS 15 & -0.01361 & 0.02728 & -0.09501 & 0.1109 & 0.03791 & 0.17647 & -0.08715 \\
\hline JCBS 39 & 0.21319 & 0.05671 & 0.18596 & 0.04633 & 0.12117 & -0.16607 & 0.03272 \\
\hline JCBS 32 & 0.20133 & 0.11511 & 0.08742 & 0.0111 & 0.15387 & -0.48421 & 0.02026 \\
\hline JCBS 83 & -0.0253 & 0.24312 & -0.11168 & 0.20914 & 0.001 & -0.06525 & 0.50581 \\
\hline JCBS 71 & -0.16415 & 0.36606 & 0.01562 & 0.30324 & -0.09696 & -0.00908 & 0.51968 \\
\hline JCBS 82 & 0.18439 & 0.19497 & 0.19233 & -0.12583 & 0.11283 & -0.13636 & 0.38774 \\
\hline JCBS 60 & 0.25375 & 0.02935 & 0.01432 & 0.08277 & 0.10098 & -0.13155 & 0.36704 \\
\hline JCBS 69 & 0.23954 & 0.39511 & 0.28687 & 0.08939 & 0.07815 & 0.01352 & 0.39382 \\
\hline JCBS 70 & 0.40468 & 0.30484 & 0.20857 & -0.09125 & 0.22714 & -0.10205 & 0.37923 \\
\hline JCBS 115 & 0.02126 & 0.07131 & 0.11341 & -0.08057 & 0.22347 & -0.14141 & 0.29409 \\
\hline JCBS 103 & 0.12435 & -0.00423 & 0.0897 & -0.0369 & 0.14394 & -0.10055 & 0.25367 \\
\hline JCBS 101 & -0.02303 & 0.14205 & 0.03216 & 0.23899 & -0.16747 & -0.06186 & 0.26872 \\
\hline JCBS 51 & 0.31593 & 0.14067 & 0.32535 & 0.10465 & 0.23092 & -0.18426 & -0.20066 \\
\hline JCBS 6 & -0.13144 & 0.10375 & 0.05389 & 0.11223 & -0.11872 & -0.17435 & -0.27017 \\
\hline JCBS 3 & 0.26648 & 0.04012 & 0.19152 & 0.11079 & 0.03446 & -0.10922 & -0.31547 \\
\hline JCBS 13 & 0.26072 & 0.20354 & 0.21544 & 0.10559 & 0.04976 & -0.09524 & -0.38319 \\
\hline JCBS 63 & 0.08246 & 0.08367 & 0.03838 & -0.01768 & 0.14894 & -0.08197 & -0.43729 \\
\hline $\begin{array}{l}\text { Un-weighted } \\
\text { explained variance }\end{array}$ & 6.1660478 & 5.4356435 & 4.6859952 & 4.0632515 & 3.5970369 & 3.2892574 & 3.4035135 \\
\hline
\end{tabular}

JCBS = Japanese Coronary-prone Behaviour Scale.

disease among Japanese men [7]. In our study, neither JAS Type A score nor any other subscale score was associated with CAD prognosis. However, our participants had jobcentred lifestyles, social dominance, and suppressed overt Type A behaviour in the previous JCBS study [5], and did not have any difference in JAS Factor $\mathrm{S}$ scores (Speed and impatience scale score) from male participants without CAD. This could be caused by the difference in measurement of speed and impatience, since the JAS Factor S score is calculated from answers to several items, but impatience in the Japan Public Health Centre-based prospective Study [7] was assessed from a single item answer.

The fourth factor of the JCBS contains questions about the Japanese mentality, such as 'You are always worried about what others think of you' (JC50), 'You put a good face on your elders and betters' (JC55), 'You often sacrifice yourself for others (JC75)', 'You think much of the public 
Table 3 Cox hazards regression analysis of coronary events with JCBS factors and standard coronary risks

\begin{tabular}{lll}
\hline Variable & Hazard ratio $(\mathbf{9 5} \% \mathrm{Cl})$ & $\boldsymbol{p}$ value \\
\hline Forced entry & $0.99(0.90-1.09)$ & 0.85 \\
Age & $0.98(0.95-1.01)$ & 0.14 \\
LDL cholesterol & $1.00(1.00-1.01)$ & 0.49 \\
Triglycerides & $0.86(0.76-0.98)$ & 0.02 \\
HDL cholesterol & $1.12(0.79-1.59)$ & 0.52 \\
Body mass index & $0.53(0.06-4.41)$ & 0.55 \\
Diabetes mellitus & $0.20(0.04-1.04)$ & 0.06 \\
Hypertension & $1.50(0.26-8.52)$ & 0.67 \\
Smoking habits & $2.61(0.86-7.84)$ & 0.09 \\
Number of coronary artery & & \\
branches involved & & 0.01 \\
Selected & $0.21(0.06-0.71)$ & \\
Factor 4 & & \\
\hline JAS J J & &
\end{tabular}

JAS = Jenkins Activity Survey, HDL = high-density lipoprotein, LDL = low-density lipoprotein, and JCBS = Japanese Coronary-prone Behaviour Scale. 'Event' includes cardiac deaths and new-onset of AMl or angina, $\mathrm{PCl}$, and CABG

image' (JC49), and so on. We called the fourth factor the Japanese spirit of 'Wa' ('harmonious groupism'), thought to be a Japanese traditional attitude used to keep order in hierarchically organized social relationships in communities and groups. 'Wa' represents a way of living that integrates a person into his or her community or group.

Some prospective studies in Western countries have implicated emotional distress, depression [8-13], and lack of social support [14-24] as risk factors for CAD prognosis. In Japan, the Osaka Acute Coronary Insufficiency Study showed that symptoms of depression were related to cardiovascular events $[25,26]$. The Shizuoka elderly cohort study showed that more positive individual perceptions of community cohesion are associated with reduced risks of cardiovascular disease, pulmonary disease, and all other causes of mortality [27]. In our study, the third factor of the JCBS was thought to be an index of social support because it contains items about family or friends, such as 'You often talk together with your family' (JC90), 'Your family listens to your problems' (JC91), 'Your friends listen to your problems' (JC92), and so on. It was not a significant factor. This could mean that the third factor of the JCBS was not the same as the structural or functional social support [28] for which a significant relationship to CAD has been reported.

This study has some limitations. First, the mechanism that explains the relationship between CAD prognosis and the Japanese spirit of ' $\mathrm{Wa}$ ' is not well known. Chronic stress and affective disorders are thought to have pathophysiological mechanisms that promote atherosclerosis [29]. The Japanese spirit of 'Wa' might have similar mechanisms. Second, nine medical centres collaborated in this study, which included community-based and intensive medical centres in different regions. This could have affected the accessibility of follow-up data. We analysed data stratified by centres to decrease this bias.

\section{Conclusion}

The Japanese spirit of 'Wa' may have protective effects against cardiovascular events among Japanese men with established CAD.

\section{Abbreviations \\ AMI: Acute myocardial infarction; CABG: Coronary artery bypass graft; CAD: Cardiovascular disease; CAG: Coronary angiography; ECGS: Eastern Collaborative Group Study; JAS: Jenkins Activity Survey; JCBS: Japanese Coronary-prone Behavior Scale; PCI: Percutaneous coronary intervention.}

\section{Competing interests}

The authors declare that they have no competing interests.

\section{Authors' contributions}

$\mathrm{RH}$ is the guarantor of the manuscript, and takes responsibility for the integrity of the data and the accuracy of the data analysis. $\mathrm{RH}, \mathrm{JH}$, and FK contributed to the study design; collection, analysis, and interpretation of data; and drafting and critical review of the manuscript. KK and NS contributed to the study design, collection and interpretation of data, and critical review of the manuscript. All authors have read and approved the final manuscript.

\section{Acknowledgments}

This study was supported by a Grant-in-Aid for Scientific Research (C) (No. 14570309) from the Japan Society for the Promotion of Science. The authors thank Hirokazu Monou, M.D., who was a main member of ECGS and contributed to study design, and Akira Yamashina, M.D. (Second Department of Internal Medicine, Tokyo Medical University), Yoshikazu Nagai, M.D. (Hachioji Medical Center of Tokyo Medical University), Kei Kitamura, M.D. (Minami Coop Hospital), Shigeru Oshima, M.D. (Division of Cardiology, Gunma Prefectural Cardiovascular Centre), Shinji Okubo, M.D. (Department of Cardiology, Kanazawa Medical University), and Keiichi Murata, M.D. (Warabi Municipal Hospital), who supported our data collection.

\section{Author details}

'Department of Health and Psychosocial Medicine, Aichi Medical University School of Medicine, 1-1 Yazako Karimata, Nagakute, Aichi 480-1195, Japan. ${ }^{2}$ Nagoya City University Graduate School of Medical Sciences and Medical School, Nagoya, Japan. ${ }^{3}$ Kimura Clinic, Kouka, Japan. ${ }^{4}$ Shinjyuku Mitui Clinic, Tokyo, Japan.

Received: 17 March 2014 Accepted: 12 January 2015

Published online: 17 January 2015

\section{References}

1. Jenkins CD, Zyzanski SJ, Rosenman RH. Progress toward validation of a computer-scored test for the type A coronary-prone behavior pattern. Psychosom Med. 1971;33(3):193-202.

2. Gulliksson M, Burell G, Vessby B, Lundin L, Toss H, Svardsudd K. Randomized controlled trial of cognitive behavioral therapy vs standard treatment to prevent recurrent cardiovascular events in patients with coronary heart disease: Secondary Prevention in Uppsala Primary Health Care project (SUPRIM). Arch Intern Med. 2011:171(2):134-40.

3. Rosengren A, Hawken S, Ounpuu S, Sliwa K, Zubaid M, Almahmeed WA, et al. Association of psychosocial risk factors with risk of acute myocardial infarction in 11119 cases and 13648 controls from 52 countries (the INTERHEART study): case-control study. Lancet. 2004;364(9438):953-62.

4. Monou H, Kimura K, Hayano J, Hosaka T, Shibata N. Deveropment of a New Scale (JCBS) assessing Coronary-Prone Behavior Pattern among Japanese. Type A. (in Japanese) 1990;1(1):19-29. 
5. Hayano J, Kimura K, Hosaka T, Shibata N, Fukunishi I, Yamasaki K, et al. Coronary disease-prone behavior among Japanese men: job-centered lifestyle and social dominance. Type A Behavior Pattern Conference. Am Heart J. 1997;134(6):1029-36.

6. Hori R, Hayano J, Monou H, Kimura K, Tsuboi H, Kamiya T, et al. Coronaryprone behavior among Japanese men. Circ J. 2003:67(2):129-32.

7. Ikeda A, Iso H, Kawachi I, Inoue M, Tsugane S, JPHC Study Group. Type A behaviour and risk of coronary heart disease: the JPHC Study. Int J Epidemiol. 2008;37(6):1395-405.

8. Carney RM, Rich MW, Freedland KE, Saini J, teVelde A, Simeone C, et al. Major depressive disorder predicts cardiac events in patients with coronary artery disease. Psychosom Med. 1988;50(6):627-33.

9. Frasure-Smith N, Lesperance F, Talajic M. Depression and 18-month prognosis after myocardial infarction. Circulation. 1995;91(4):999-1005.

10. Barefoot JC, Helms MJ, Mark DB, Blumenthal JA, Califf RM, Haney TL, et al. Depression and long-term mortality risk in patients with coronary artery disease. Am J Cardiol. 1996;78(6):613-7.

11. Denollet J, Brutsaert DL. Personality, disease severity, and the risk of long-term cardiac events in patients with a decreased ejection fraction after myocardial infarction. Circulation. 1998;97(2):167-73.

12. Frasure-Smith $N$, Lesperance $F$, Juneau M, Talajic M, Bourassa MG. Gender, depression, and one-year prognosis after myocardial infarction. Psychosom Med. 1999;61(1):26-37.

13. Frasure-Smith N, Lesperance F, Gravel G, Masson A, Juneau M, Talajic M, et al. Social support, depression, and mortality during the first year after myocardial infarction. Circulation. 2000;101(16):1919-24.

14. Chandra V, Szklo M, Goldberg R, Tonascia J. The impact of marital status on survival after an acute myocardial infarction: a population-based study. Am J Epidemiol. 1983;117(3):320-5.

15. Ruberman W, Weinblatt E, Goldberg JD, Chaudhary BS. Psychosocial influences on mortality after myocardial infarction. N Engl J Med. 1984;311 (9):552-9.

16. Wiklund I, Oden A, Sanne H, Ulvenstam G, Wilhelmsson C, Wilhelmsen L. Prognostic importance of somatic and psychosocial variables after a first myocardial infarction. Am J Epidemiol. 1988;128(4):786-95.

17. Ahern DK, Gorkin L, Anderson JL, Tierney C, Hallstrom A, Ewart C, et al. Biobehavioral variables and mortality or cardiac arrest in the Cardiac Arrhythmia Pilot Study (CAPS). Am J Cardiol. 1990;66(1):59-62.

18. Case RB, Moss AJ, Case N, McDermott M, Eberly S. Living alone after myocardial infarction. Impact on prognosis. JAMA. 1992;267(4):515-9.

19. Williams RB, Barefoot JC, Califf RM, Haney TL, Saunders WB, Pryor DB, et al. Prognostic importance of social and economic resources among medically treated patients with angiographically documented coronary artery disease. JAMA. 1992;267(4):520-4.

20. Berkman LF, Leo-Summers L, Horwitz Rl. Emotional support and survival after myocardial infarction. A prospective, population-based study of the elderly. Ann Intern Med. 1992;117(12):1003-9.

21. Gorkin L, Schron EB, Brooks MM, Wiklund I, Kellen J, Verter J, et al. Psychosocial predictors of mortality in the Cardiac Arrhythmia Suppression Trial-1 (CAST-1). Am J Cardiol. 1993;71(4):263-7.

22. Jenkinson CM, Madeley RJ, Mitchell JR, Turner ID. The influence of psychosocial factors on survival after myocardial infarction. Public Health. 1993;107(5):305-17.

23. Woloshin S, Schwartz LM, Tosteson AN, Chang CH, Wright B, Plohman J, et al. Perceived adequacy of tangible social support and health outcomes in patients with coronary artery disease. J Gen Intern Med. 1997;12(10):613-8.

24. Barth J, Schneider S, von Kanel R. Lack of social support in the etiology and the prognosis of coronary heart disease: a systematic review and metaanalysis. Psychosom Med. 2010;72(3):229-38.

25. Shiotani I, Sato H, Kinjo K, Nakatani D, Mizuno H, Ohnishi Y, et al. Depressive symptoms predict 12-month prognosis in elderly patients with acute myocardial infarction. J Cardiovasc Risk. 2002;9(3):153-60.

26. Shiozaki M, Iso H, Ohira T, Nakatani D, Shimizu M, Sakata $Y$, et al. Longitudinal risk of cardiovascular events in relation to depression symptoms after discharge among survivors of myocardial infarction. Osaka Acute Coronary Insufficiency Study. Circ J. 2011;75(12):2878-84.

27. Inoue S, Yorifuji T, Takao S, Doi H, Kawachi I. Social cohesion and mortality: a survival analysis of older adults in Japan. Am J Public Health. 2013;103(12):e60. -6.
28. Lett HS, Blumenthal JA, Babyak MA, Strauman TJ, Robins C, Sherwood A. Social support and coronary heart disease: epidemiologic evidence and implications for treatment. Psychosom Med. 2005;67(6):869-78.

29. Rozanski A, Blumenthal JA, Davidson KW, Saab PG, Kubzansky L. The epidemiology, pathophysiology, and management of psychosocial risk factors in cardiac practice: the emerging field of behavioral cardiology. J Am Coll Cardiol. 2005;45(5):637-51.

\section{Submit your next manuscript to BioMed Central and take full advantage of:}

- Convenient online submission

- Thorough peer review

- No space constraints or color figure charges

- Immediate publication on acceptance

- Inclusion in PubMed, CAS, Scopus and Google Scholar

- Research which is freely available for redistribution 